\title{
Two-weight and three-weight linear codes based on Weil sums
}

\author{
Gaopeng Jian
}

\begin{abstract}
Linear codes with few weights have applications in secret sharing, authentication codes, association schemes and strongly regular graphs. In this paper, several classes of two-weight and three-weight linear codes are presented and their weight distributions are determined using Weil sums. Some of the linear codes obtained are optimal or almost optimal with respect to the Griesmer bound.
\end{abstract}

Keywords Linear codes . Weight distribution . Weil sums

\section{Introduction}

Let $\mathbb{F}_{p}$ be the finite field with $p$ elements, where $p$ is an odd prime. An $[n, k, d]$ linear code $C$ over $\mathbb{F}_{p}$ is a $k$-dimensional subspace of $\mathbb{F}_{p}^{n}$ with minimum Hamming distance $d$. Let $A_{i}$ be the number of codewords with Hamming weight $i$ in $C$. The polynomial $1+A_{1} z+A_{2} z^{2}+\cdots+A_{n} z^{n}$ is called the weight enumerator of $C$ and the sequence $\left(1, A_{1}, A_{2}, \ldots, A_{n}\right)$ called the weight distribution of $C$. If the number of nonzero $A_{i}$ in the sequence $\left(A_{1}, A_{2}, \ldots, A_{n}\right)$ is equal to $t$, we call $C$ a $t$-weight code. The weight distribution of a code contains important information on its error correcting capability and the error probability of its error detection and correction with respect to some algorithms [20. In addition, much attention has been paid to two-weight and three-weight linear codes [19,22, 14, 12, 11, 13, 18, 8, 9] due to their applications in secret sharing [21,3], strongly regular graphs [2], association schemes 1 and authentication codes 7 .

Let $\operatorname{Tr}$ denote the trace function from $\mathbb{F}_{q}$ to $\mathbb{F}_{p}$. For a set $D=\left\{d_{1}, d_{2}, \ldots, d_{n}\right\} \subset$ $\mathbb{F}_{q}$, define a $p$-ary linear code of length $n$ by

$$
C_{D}=\left\{c(a)=\left(\operatorname{Tr}\left(a d_{1}\right), \operatorname{Tr}\left(a d_{2}\right), \ldots, \operatorname{Tr}\left(a d_{n}\right)\right): a \in \mathbb{F}_{q}\right\}
$$

The set $D$ is called the defining set of $C_{D}$. The construction was first proposed by Ding et al. in [6] and many classes of known codes could be produced by properly selecting the defining set.

Gaopeng Jian

Key Laboratory of Machine Perception(MOE), School of EECS, Peking University, Beijing 100871, P.R.China;

E-mail: gpjian@pku.edu.cn 
Motivated by the above construction, Li et al. [16] defined a $p$-ary linear code by

$$
C_{D}=\left\{c(a, b)=(\operatorname{Tr}(a x+b y))_{(x, y) \in D}: a, b \in \mathbb{F}_{q}\right\}
$$

where $D \subset \mathbb{F}_{q}^{2}$ is also called a defining set. It can be viewed as a generalization of cyclic codes whose duals have two zeros. In this paper, we extend their results by choosing different defining sets given by

$$
D_{1}=\left\{(x, y) \in \mathbb{F}_{q}^{2} \backslash\{(0,0)\}: \operatorname{Tr}\left(x+y^{p^{u}+1}\right)=0\right\}
$$

and

$$
D_{2}=\left\{(x, y) \in \mathbb{F}_{q}^{2} \backslash\{(0,0)\}: \operatorname{Tr}\left(x^{2}+y^{p^{u}+1}\right)=0\right\} .
$$

We mainly use Weil sums to determine their parameters and weight distributions. In particular, some of the linear codes obtained are optimal or almost optimal with respect to the Griesmer bound 10 .

The rest of this paper is organized as follows. In section 2 we introduce some basic notations and results on group characters, character sums and Pless power moments, which will be employed later. In section 3 we present the parameters of several classes of two-weight and three-weight linear codes. We also give some examples. Section 4 is devoted to giving the proofs of main results. Section 5 summarizes this paper.

\section{Preliminaries}

From now on we fix the following notations.

$-q=p^{m}$, where $p$ is an odd prime and $m$ is a positive integer.

$-s=\frac{m}{2}$.

- $u$ is a positive integer, $v=\operatorname{gcd}(m, u)$.

- Tr is the trace function from $\mathbb{F}_{q}$ to $\mathbb{F}_{p}$.

$-\zeta_{p}=e^{\frac{2 \pi \sqrt{-1}}{p}}$ is the primitive $p$-th root of unity.

- $\eta$ and $\eta_{p}$ are the quadratic multiplicative characters of $\mathbb{F}_{q}$ and $\mathbb{F}_{p}$, respectively

\subsection{Group characters and Gauss sums}

An additive character of $\mathbb{F}_{q}$ is a homomorphism from the additive group $\mathbb{F}_{q}$ into the multiplicative group composed by the $p$-th roots of unity in the complex numbers. For each $b \in \mathbb{F}_{q}$, the function

$$
\chi_{b}(x)=\zeta_{p}^{\operatorname{Tr}(b x)} \text { for all } x \in \mathbb{F}_{q}
$$

defines an additive character of $\mathbb{F}_{q}$. It is clear that $\chi_{0}(x)=1$ for all $x \in \mathbb{F}_{q}$ and it is called the trivial additive character of $\mathbb{F}_{q}$. The character $\chi:=\chi_{1}$ is called the canonical additive character of $\mathbb{F}_{q}$. It is easy to see that $\chi_{b}(x)=\chi(b x)$ for all $b, x \in \mathbb{F}_{q}$. The orthogonal property of additive characters is given by

$$
\sum_{x \in \mathbb{F}_{q}} \chi_{b}(x)= \begin{cases}q, & \text { if } b=0 \\ 0, & \text { otherwise. }\end{cases}
$$


A multiplicative character of $\mathbb{F}_{q}$ is a homomorphism from the multiplicative group $\mathbb{F}_{q}^{*}=\mathbb{F}_{q} \backslash\{0\}$ into the multiplicative group composed by the $(q-1)$-th roots of unity in the complex numbers. Let $\lambda$ be a multiplicative character of $\mathbb{F}_{q}$, we define the Gauss sum over $\mathbb{F}_{q}$ by

$$
G(\lambda)=\sum_{x \in \mathbb{F}_{q}^{*}} \lambda(x) \chi(x)
$$

The explicit value of Gauss sums are very difficult to determine and are known for only a few cases. For future use, we state some results about Gauss sums. The quadratic Gauss sums are known and given in the following lemma.

Lemma 1 ([17, Theorem 5.15])

$$
G(\eta)=(-1)^{m-1} \sqrt{\left(p^{*}\right)^{m}}=\left\{\begin{array}{lll}
(-1)^{m-1} \sqrt{q}, & \text { if } p \equiv 1 \quad(\bmod 4) \\
(-1)^{m-1}(\sqrt{-1})^{m} \sqrt{q}, & \text { if } p \equiv 3 \quad(\bmod 4)
\end{array}\right.
$$

where $p^{*}=(-1)^{\frac{p-1}{2}} p$.

Lemma $2\left(\left[\right.\right.$, Lemma 7]) For $x \in \mathbb{F}_{p}^{*}$,

$$
\eta(x)= \begin{cases}1, & \text { if } m \text { is even } \\ \eta_{p}(x), & \text { if } m \text { is odd }\end{cases}
$$

\subsection{Weil sums}

Weil sums are defined by $\sum_{x \in \mathbb{F}_{q}} \chi(f(x))$ where $f(x) \in \mathbb{F}_{q}[X]$. In [4, 5], Coulter evaluated some Weil sums given by

$$
S_{u}(a, b)=\sum_{x \in \mathbb{F}_{q}} \chi\left(a x^{p^{u}+1}+b x\right), a \in \mathbb{F}_{q}^{*}, b \in \mathbb{F}_{q} .
$$

Lemma 3 If $\frac{m}{v}$ be odd, then

$$
S_{u}(a, 0)=G(\eta) \eta(a)=\left\{\begin{array}{lll}
(-1)^{m-1} \sqrt{q} \eta(a), & \text { if } p \equiv 1 \quad(\bmod 4) \\
(-1)^{m-1}(\sqrt{-1})^{m} \sqrt{q} \eta(a), & \text { if } p \equiv 3 \quad(\bmod 4)
\end{array}\right.
$$

Lemma 4 If $\frac{m}{v}$ is even, then

$$
S_{u}(a, 0)= \begin{cases}p^{s}, & \text { if } \frac{s}{v} \text { is even and } a^{\frac{q-1}{p^{v}+1}} \neq(-1)^{\frac{s}{v}}, \\ -p^{s+v}, & \text { if } \frac{s}{v} \text { is even and } a^{\frac{q-1}{p^{v}+1}}=(-1)^{\frac{s}{v}}, \\ -p^{s}, & \text { if } \frac{s}{v} \text { is odd and } a^{\frac{q-1}{p^{v}+1}} \neq(-1)^{\frac{s}{v}}, \\ p^{s+v}, & \text { if } \frac{s}{v} \text { is odd and } a^{\frac{q-1}{p^{v}+1}}=(-1)^{\frac{s}{v}} .\end{cases}
$$

Lemma 5 The equation

$$
a^{p^{u}} X^{p^{2 u}}+a X=0
$$

is solvable over $\mathbb{F}_{q}^{*}$ if and only if $\frac{m}{v}$ is even and $a^{\frac{q-1}{p^{v}+1}}=(-1)^{\frac{s}{v}}$. In such cases there are $p^{2 v}-1$ non-zero solutions. 
Lemma 6 Suppose $f(X)=a^{p^{u}} X^{p^{2 u}}+a X$ is a permutation polynomial over $\mathbb{F}_{q}$. Let $x_{0}$ be the unique solution of the equation $f(X)=-b^{p^{u}}$. The evaluation of $S_{u}(a, b)$ partitions into the following two cases:

1. If $\frac{m}{v}$ is odd, then

$$
\begin{aligned}
S_{u}(a, b) & =G(\eta) \eta(a) \bar{\chi}\left(a x_{0}^{p^{u}+1}\right) \\
& =\left\{\begin{array}{lll}
(-1)^{m-1} \sqrt{q} \eta(a) \bar{\chi}\left(a x_{0}^{p^{u}+1}\right), & \text { if } p \equiv 1 & (\bmod 4), \\
(-1)^{m-1}(\sqrt{-1})^{m} \sqrt{q} \eta(a) \bar{\chi}\left(a x_{0}^{p^{u}+1}\right), & \text { if } p \equiv 3 & (\bmod 4) .
\end{array}\right.
\end{aligned}
$$

2. If $\frac{m}{v}$ is even, then $a^{\frac{q-1}{p^{v}+1}} \neq(-1)^{\frac{s}{v}}$ and

$$
S_{u}(a, b)=(-1)^{\frac{s}{v}} p^{s} \bar{\chi}\left(a x_{0}^{p^{u}+1}\right)
$$

Lemma 7 Suppose $f(X)=a^{p^{u}} X^{p^{2 u}}+a X$ is not a permutation polynomial over $\mathbb{F}_{q}$, then $S_{u}(a, b)=0$ unless the equation $f(X)=-b^{p^{u}}$ is solvable. If the equation is solvable, with some solution $x_{0}$ say, then

$$
S_{u}(a, b)=-(-1)^{\frac{s}{v}} p^{s+v} \bar{\chi}\left(a x_{0}^{p^{u}+1}\right) .
$$

The following lemma is a special case $(u=0)$ of Lemma 3 and 6 and is also proved in [17, Theorem 5.33].

\section{Lemma 8}

$$
Q(a, b)=\sum_{x \in \mathbb{F}_{q}} \chi\left(a x^{2}+b x\right)=G(\eta) \eta(a) \bar{\chi}\left(\frac{b^{2}}{4 a}\right), a \in \mathbb{F}_{q}^{*}, b \in \mathbb{F}_{q}
$$

\subsection{The Pless power moments}

For an $[n, k, d]$ code $C$ over $\mathbb{F}_{p}$ with weight distribution $\left(1, A_{1}, \ldots, A_{n}\right)$, we denote by $\left(1, A_{1}^{\perp}, \ldots, A_{n}^{\perp}\right)$ the weight distribution of its dual code. The first two Pless power moments are given as follows [15, p.259]:

$$
\begin{gathered}
\sum_{j=0}^{n} A_{j}=p^{k}, \\
\sum_{j=0}^{n} j A_{j}=p^{k-1}\left(p n-n-A_{1}^{\perp}\right) .
\end{gathered}
$$

For the code $C_{D}$ defined by (11), $A_{1}^{\perp}=0$ if $(0,0) \notin D$ by the nondegenerate property of the trace function. 
Table 1: The weight distribution of $C_{D_{1}}$ if $m$ is odd

\begin{tabular}{ll}
\hline Weight $w$ & Multiplicity $A_{w}$ \\
\hline 0 & 1 \\
$(p-1) p^{2 m-2}$ & $p^{2 m}-1-(p-1)^{2} p^{m-1}$ \\
$(p-1) p^{2 m-2}\left(1-\frac{1}{(p-1) p^{\frac{m-1}{2}}}\right)$ & $\frac{1}{2}(p-1)^{2}\left(p^{m-1}+p^{\frac{m-1}{2}}\right)$ \\
$(p-1) p^{2 m-2}\left(1+\frac{1}{(p-1) p^{\frac{m-1}{2}}}\right)$ & $\frac{1}{2}(p-1)^{2}\left(p^{m-1}-p^{\frac{m-1}{2}}\right)$ \\
\hline
\end{tabular}

Table 2: The weight distribution of $C_{D_{1}}$ if $\frac{m}{v}$ is odd and $v$ is even

\begin{tabular}{ll}
\hline Weight $w$ & Multiplicity $A_{w}$ \\
\hline 0 & 1 \\
$(p-1) p^{2 m-2}$ & $p^{2 m}-1-(p-1) p^{m}$ \\
$(p-1) p^{2 m-2}\left(1-\frac{G(\eta)}{q}\right)$ & $(p-1) p^{m-1}\left(1+\frac{(p-1) G(\eta)}{q}\right)$ \\
$(p-1) p^{2 m-2}\left(1+\frac{G(\eta)}{(p-1) q}\right)$ & $(p-1) p^{m-1}\left(p-1-\frac{(p-1) G(\eta)}{q}\right)$ \\
\hline
\end{tabular}

Table 3: The weight distribution of $C_{D_{1}}$ if $\frac{m}{v} \equiv 2(\bmod 4)$

\begin{tabular}{ll}
\hline Weight $w$ & Multiplicity $A_{w}$ \\
\hline 0 & 1 \\
$(p-1) p^{2 m-2}$ & $p^{2 m}-1-(p-1) p^{m}$ \\
$(p-1) p^{2 m-2}\left(1+\frac{1}{p^{s}}\right)$ & $(p-1)\left(p^{m-1}-p^{s}+p^{s-1}\right)$ \\
$(p-1) p^{2 m-2}\left(1-\frac{1}{(p-1) p^{s}}\right)$ & $(p-1)\left(p^{s}+1\right)\left(p^{s}-p^{s-1}\right)$ \\
\hline
\end{tabular}

\section{The statements of main results}

Theorem 1 Let $C_{D_{1}}$ be defined by (11), where $D_{1}$ is defined in (2). If $m$ is odd, then $C_{D_{1}}$ is a $\left[p^{2 m-1}-1,2 m\right]$ three-weight linear code with weight distribution given in Table 1 .

Example 1 Let $p=3$ and $m=3$. Then the code $C_{D_{1}}$ has parameters [242,6,135] and weight enumerator $1+24 z^{135}+692 z^{162}+12 z^{189}$.

Theorem 2 Let $C_{D_{1}}$ be defined by (1), where $D_{1}$ is defined in (2). If $\frac{m}{v}$ is odd and $v$ is even, then $C_{D_{1}}$ is a $\left[p^{2 m-1}-1,2 m\right]$ three-weight linear code with weight distribution given in Table 2

Example 2 Let $p=3, m=2$ and $u=4$. Then $v=2, \frac{m}{v}=1$ and $G(\eta)=3$. The code $C_{D_{1}}$ has parameters $[26,4,12]$ and weight enumerator $1+10 z^{12}+62 z^{18}+8 z^{21}$.

Theorem 3 Let $C_{D_{1}}$ be defined by (11), where $D_{1}$ is defined in (2). If $\frac{m}{v} \equiv 2(\bmod 4)$, then $C_{D_{1}}$ is a $\left[p^{2 m-1}-1,2 m\right]$ three-weight linear code with weight distribution given in Table 3 .

Example 3 Let $p=3, m=2$ and $u=3$. Then $v=1, \frac{m}{v}=2$ and $s=1$. The code $C_{D_{1}}$ has parameters $[26,4,15]$ and weight enumerator $1+16 z^{15}+62 z^{18}+2 z^{24}$. 
Table 4: The weight distribution of $C_{D_{1}}$ if $\frac{m}{v} \equiv 0(\bmod 4)$

\begin{tabular}{ll}
\hline Weight $w$ & Multiplicity $A_{w}$ \\
\hline 0 & 1 \\
$(p-1) p^{2 m-2}$ & $p^{2 m}-1-(p-1) p^{m-2 v}$ \\
$(p-1) p^{2 m-2}\left(1+\frac{1}{p^{s-v}}\right)$ & $(p-1)\left(p^{m-2 v-1}-p^{s-v}+p^{s-v-1}\right)$ \\
$(p-1) p^{2 m-2}\left(1-\frac{1}{(p-1) p^{s-v}}\right)$ & $(p-1)\left(p^{s-v}+1\right)\left(p^{s-v}-p^{s-v-1}\right)$ \\
\hline
\end{tabular}

Table 5: The weight distribution of $C_{D_{2}}$ if $\frac{m}{v}$ is odd or $\frac{m}{v} \equiv 2(\bmod 4)$

(a) $p \equiv 3(\bmod 4)$ and $v$ is odd

\begin{tabular}{ll}
\hline Weight $w$ & Multiplicity $A_{w}$ \\
\hline 0 & 1 \\
$(p-1) p^{2 m-2}$ & $\left(p^{m}+1\right)\left(p^{m-1}-1\right)$ \\
$(p-1) p^{2 m-2}\left(1-\frac{1}{p^{m-1}}\right)$ & $\left(p^{m}+1\right) p^{m-1}(p-1)$ \\
\hline
\end{tabular}

(b) otherwise

\begin{tabular}{ll}
\hline Weight $w$ & Multiplicity $A_{w}$ \\
\hline 0 & 1 \\
$(p-1) p^{2 m-2}$ & $\left(p^{m}-1\right)\left(p^{m-1}+1\right)$ \\
$(p-1) p^{2 m-2}\left(1+\frac{1}{p^{m-1}}\right)$ & $\left(p^{m}-1\right) p^{m-1}(p-1)$ \\
\hline
\end{tabular}

Theorem 4 Let $C_{D_{1}}$ be defined by (11), where $D_{1}$ is defined in (2). If $\frac{m}{v} \equiv 0(\bmod 4)$, then $C_{D_{1}}$ is a $\left[p^{2 m-1}-1,2 m\right]$ three-weight linear code with weight distribution given in Table 4 .

Example 4 Let $p=3, m=4$ and $u=3$. Then $v=1, \frac{m}{v}=4$ and $s=2$. The code $C_{D_{1}}$ has parameters [2186,8,1215] and weight enumerator $1+16 z^{1215}+6542 z^{1458}+$ $2 z^{1944}$.

Theorem 5 Let $C_{D_{2}}$ be defined by (1), where $D_{2}$ is defined in (3). If $\frac{m}{v}$ is odd or $\frac{m}{v} \equiv 2(\bmod 4)$, then $C_{D_{2}}$ is an $[n, 2 m]$ two-weight linear code with weight distribution given in Table 5, where

$$
n= \begin{cases}\left(p^{m}+1\right)\left(p^{m-1}-1\right), & \text { if } p \equiv 3 \quad(\bmod 4) \text { and } v \text { is odd } \\ \left(p^{m}-1\right)\left(p^{m-1}+1\right), & \text { otherwise }\end{cases}
$$

Note that for any $a \in \mathbb{F}_{p}^{*}, \operatorname{Tr}\left((a x)^{2}+(a y)^{p^{u}+1}\right)=a^{2} \operatorname{Tr}\left(x^{2}+y^{p^{u}+1}\right)$. Then we can select a subset $\overline{D_{2}}$ of $D_{2}$ such that $\bigcup_{a \in \mathbb{F}_{p}^{*}} a \overline{D_{2}}$ is a partition of $D_{2}$. Hence, the code $C_{D_{2}}$ can be punctured into a shorter linear codes $C_{\overline{D_{2}}}$ whose weights can be obtained from the original $C_{D_{2}}$ by dividing the common divisor $p-1$.

Example 5 Let $p=3$ and $m=3$. Then the code $C_{D_{2}}$ has parameters [224,6,144] and weight enumerator $1+504 z^{144}+224 z^{162}$. The code $C_{\overline{D_{2}}}$ has parameters [112,6,72] and weight enumerator $1+504 z^{72}+224 z^{81}$, which is almost optimal as the best linear code of length 112 and dimension 6 over $\mathbb{F}_{3}$ has minimum weight 73 according to the Griesmer bound. 
Table 6: The weight distribution of $C_{D_{2}}$ if $\frac{m}{v} \equiv 0(\bmod 4)$

\begin{tabular}{ll}
\hline Weight $w$ & Multiplicity $A_{w}$ \\
\hline 0 & 1 \\
$(p-1) p^{2 m-2}$ & $\left(p^{m-v}-1\right)\left(p^{m-v-1}+1\right)$ \\
$(p-1) p^{2 m-2}\left(1+\frac{p-1}{p^{m-v}}\right)$ & $p^{2 m}-p^{2 m-2 v}$ \\
$(p-1) p^{2 m-2}\left(1+\frac{1}{p^{m-v-1}}\right)$ & $\left(p^{m-v}-1\right) p^{m-v-1}(p-1)$ \\
\hline
\end{tabular}

Example 6 Let $p=3, m=2$ and $u=3$. Then $v=1$ and $\frac{m}{v}=2$. The code $C_{D_{2}}$ has parameters $[20,4,12]$ and weight enumerator $1+60 z^{12}+20 z^{18}$. The code $C_{\overline{D_{2}}}$ has parameters $[10,4,6]$ and weight enumerator $1+60 z^{6}+20 z^{9}$. Both codes are optimal according to the Griesmer bound, and $C_{D_{2}}$ is different from the best known linear codes from the Magma BKLC(GF(3),20,4) which has a different weight enumerator $1+60 z^{12}+18 z^{15}+2 z^{18}$.

Example 7 Let $p=3, m=2$ and $u=4$. Then $v=2$ and $\frac{m}{v}=1$. The code $C_{D_{2}}$ has parameters $[32,4,18]$ and weight enumerator $1+32 z^{18}+48 z^{24}$. The code $C_{\overline{D_{2}}}$ has parameters $[16,4,9]$ and weight enumerator $1+32 z^{9}+48 z^{12}$, which is optimal according to the Griesmer bound. In addition, $C_{\overline{D_{2}}}$ is different from the best known linear codes from the Magma BKLC(GF(3),16,4) which has a different weight enumerator $1+$ $50 z^{9}+30 z^{12}$.

Example 8 Let $p=3, m=4$ and $u=2$. Then $v=2$ and $\frac{m}{u}=2$. The code $C_{D_{2}}$ has parameters [2240,8,1458] and weight enumerator $1+2240 z^{1458}+4320 z^{1512}$. The code $C_{\overline{D_{2}}}$ has parameters [1120,8,729] and weight enumerator $1+2240 z^{729}+4320 z^{756}$.

Theorem 6 Let $C_{D_{2}}$ be defined by (11), where $D_{2}$ is defined in (3). If $\frac{m}{v} \equiv 0(\bmod 4)$, then $C_{D_{2}}$ is a $\left[p^{2 m-1}+p^{m+v}-p^{m+v-1}-1,2 m\right]$ three-weight linear code with weight distribution given in Table 6 .

Example 9 Let $p=3, m=4$ and $u=3$. Then $v=1, \frac{m}{v}=4$ and $s=2$. The code $C_{D_{2}}$ has parameters [2348,8,1458] and weight enumerator $1+260 z^{1458}+$ $5832 z^{1566}+468 z^{1620}$. The code $C_{\overline{D_{2}}}$ has parameters [1174,8,729] and weight enumerator $1+260 z^{729}+5832 z^{783}+468 z^{810}$.

\section{The proofs of main results}

4.1 Some auxiliary results

Lemma 9 Let

$$
n_{1}=\left|D_{1}\right|=\left|\left\{(x, y) \in \mathbb{F}_{q}^{2} \backslash\{(0,0)\}: \operatorname{Tr}\left(x+y^{p^{u}+1}\right)=0\right\}\right|
$$

Then $n_{1}=p^{2 m-1}-1$. 
Proof By the orthogonal property of additive characters

$$
\begin{aligned}
n_{1} & =\sum_{x, y \in \mathbb{F}_{q}} \frac{1}{p} \sum_{z \in \mathbb{F}_{p}} \zeta_{p}^{z \operatorname{Tr}\left(x+y^{p^{u}+1}\right)}-1 \\
& =\frac{q^{2}}{p}-1+\frac{1}{p} \sum_{z \in \mathbb{F}_{p}^{*}} \sum_{x \in \mathbb{F}_{q}} \zeta_{p}^{\operatorname{Tr}(z x)} S_{u}(z, 0) \\
& =p^{2 m-1}-1 .
\end{aligned}
$$

Lemma 10 If $\frac{m}{v}$ is even, then for $x \in \mathbb{F}_{p}^{*}$,

$$
x^{\frac{q-1}{p^{v}+1}}=1
$$

Proof Consider

$$
\frac{q-1}{\left(p^{v}+1\right)(p-1)}=\frac{\left(p^{s}-1\right)\left(p^{s}+1\right)}{\left(p^{v}+1\right)(p-1)}
$$

If $\frac{s}{v}$ is odd, then $\left(p^{v}+1\right)(p-1) \mid\left(p^{s}-1\right)\left(p^{s}+1\right)$ for $p^{v}+1 \mid p^{s}+1$ and $p-1 \mid p^{s}-1$. If $\frac{s}{v}$ is even, it's easy to see $\operatorname{gcd}\left(p-1, p^{v}+1\right)=2$. So $\left(p^{v}+1\right)(p-1) \mid\left(p^{s}-1\right)\left(p^{s}+1\right)$ for $p^{v}+1 \mid p^{s}-1$ and $p-1 \mid p^{s}-1$.

Lemma 11 Let

$$
n_{2}=\left|D_{2}\right|=\left|\left\{(x, y) \in \mathbb{F}_{q}^{2} \backslash\{(0,0)\}: \operatorname{Tr}\left(x^{2}+y^{p^{u}+1}\right)=0\right\}\right| .
$$

Then if $\frac{m}{v}$ is odd or $\frac{m}{v} \equiv 2(\bmod 4)$,

$$
n_{2}= \begin{cases}\left(p^{m}+1\right)\left(p^{m-1}-1\right), & \text { if } p \equiv 3 \quad(\bmod 4) \text { and } v \text { is odd } \\ \left(p^{m}-1\right)\left(p^{m-1}+1\right), & \text { otherwise }\end{cases}
$$

If $\frac{m}{v} \equiv 0(\bmod 4)$

$$
n_{2}=p^{2 m-1}+p^{m+v}-p^{m+v-1}-1 .
$$

Note that if $p \equiv 3(\bmod 4)$ and $m=1, D_{2}=\left\{(x, y) \in \mathbb{F}_{p}^{2} \backslash\{(0,0)\}: x^{2}+y^{2}=0\right\}=\varnothing$, so $n_{2}=0$.

Proof By the orthogonal property of additive characters

$$
\begin{aligned}
n_{2} & =\sum_{x, y \in \mathbb{F}_{q}} \frac{1}{p} \sum_{z \in \mathbb{F}_{p}} \zeta_{p}^{z \operatorname{Tr}\left(x^{2}+y^{p^{u}+1}\right)}-1 \\
& =\frac{q^{2}}{p}-1+\frac{1}{p} \sum_{z \in \mathbb{F}_{p}^{*}} Q(z, 0) S_{u}(z, 0) \\
& =p^{2 m-1}-1+\frac{1}{p} \Omega
\end{aligned}
$$

where $\Omega=\sum_{z \in \mathbb{F}_{p}^{*}} Q(z, 0) S_{u}(z, 0)$. Then we evaluate $\Omega$ through three cases. 
1. If $\frac{m}{v}$ is odd, by Lemma 1

$$
G(\eta)^{2}=\left(p^{*}\right)^{m}= \begin{cases}-p^{m}, & \text { if } p \equiv 3 \quad(\bmod 4) \text { and } v \text { is odd } \\ p^{m}, & \text { otherwise }\end{cases}
$$

By Lemma 3 and 8

$$
\begin{aligned}
\Omega & =(p-1) G(\eta)^{2} \\
& = \begin{cases}-(p-1) p^{m}, & \text { if } p \equiv 3 \quad(\bmod 4) \text { and } v \text { is odd }, \\
(p-1) p^{m}, & \text { otherwise. }\end{cases}
\end{aligned}
$$

2. If $\frac{m}{v} \equiv 2(\bmod 4)$, by Lemma 1

$$
G(\eta)=-\left(p^{*}\right)^{s}= \begin{cases}p^{s}, & \text { if } p \equiv 3 \quad(\bmod 4) \text { and } v \text { is odd } \\ -p^{s}, & \text { otherwise }\end{cases}
$$

By Lemma 2, 4, 8, and 10

$$
\begin{aligned}
\Omega & =-p^{s} G(\eta) \sum_{z_{1} \in \mathbb{F}_{p}^{*}} \eta\left(z_{1}\right) \\
& = \begin{cases}-(p-1) p^{m}, & \text { if } p \equiv 3 \quad(\bmod 4) \text { and } v \text { is odd }, \\
(p-1) p^{m}, & \text { otherwise. }\end{cases}
\end{aligned}
$$

3. If $\frac{m}{v} \equiv 0(\bmod 4)$, by Lemma 1] $G(\eta)=-p^{s}$ and by Lemma 2, 4, 8 and 10

$$
\Omega=-p^{s+v} G(\eta) \sum_{z_{1} \in \mathbb{F}_{p}^{*}} \eta\left(z_{1}\right)=(p-1) p^{m+v} .
$$

By (44),(5), (6) and (7) we complete the proof.

Lemma 12 If $m$ is odd, then

$$
B_{1}=\left|\left\{x \in \mathbb{F}_{q}: \operatorname{Tr}\left(x^{p^{u}+1}\right)=0\right\}\right|=p^{m-1} .
$$

Proof By Lemma 2 and 3

$$
\begin{aligned}
B_{1} & =\sum_{x \in \mathbb{F}_{q}} \frac{1}{p} \sum_{z \in \mathbb{F}_{p}} \zeta_{p}^{z \operatorname{Tr}\left(x^{p^{u}+1}\right)} \\
& =\frac{1}{p} \sum_{x \in \mathbb{F}_{q}}\left(1+\sum_{z \in \mathbb{F}_{p}^{*}} \zeta_{p}^{z \operatorname{Tr}\left(x^{p^{u}+1}\right)}\right) \\
& =p^{m-1}+\frac{1}{p} \sum_{z \in \mathbb{F}_{p}^{*}} S_{u}(z, 0) \\
& =p^{m-1}+\frac{G(\eta)}{p} \sum_{z \in \mathbb{F}_{p}^{*}} \eta_{p}(z) \\
& =p^{m-1}
\end{aligned}
$$


Lemma 13 If $\frac{m}{v} \equiv 0(\bmod 4)$, then

$$
B_{2}=\mid\left\{c \in \mathbb{F}_{q}: X^{p^{2 u}}+X=c^{p^{u}} \text { is solvable in } \mathbb{F}_{q}\right\} \mid=p^{m-2 v} .
$$

Proof The map

$$
x \mapsto x^{p^{2 u}}+x, x \in \mathbb{F}_{q}
$$

is $\mathbb{F}_{p}$-linear. By Lemma可, the equation $X^{p^{2 u}}+X=0$ has $p^{2 v}$ solutions in $\mathbb{F}_{q}$. Note that $X^{p^{u}}$ is a permutation polynomial over $\mathbb{F}_{q}$, so $B_{2}=p^{m-2 v}$.

\subsection{The proofs of Theorems 1, 2, 3 and 4}

By Lemma 9 the code $C_{D_{1}}$ has length $n_{1}=p^{2 m-1}-1$. For a codeword $c(a, b)$, $(a, b) \in \mathbb{F}_{q}^{2} \backslash\{(0,0)\}$, we will show that the Hamming weight $W_{H}(c(a, b))>0$, so the dimension of $C_{D_{1}}$ is $2 m$.

We begin with the following equation:

$$
X^{p^{2 u}}+X=\left(a^{-1} b\right)^{p^{u}} .
$$

By Lemma [5] it's not always solvable over $\mathbb{F}_{q}$ if $\frac{m}{v} \equiv 0(\bmod 4)$, and has an unique solution otherwise. Let $\gamma_{a, b}$ be some solution of (E1) if it exists. Note that for $a, z_{2} \in \mathbb{F}_{p}^{*}$ the equation $\left(-z_{2} a\right)^{p^{u}} X^{p^{2 u}}+\left(-z_{2} a\right) X=-\left(z_{2} b\right)^{p^{u}}$ is equivalent to (E1). For $(a, b) \in \mathbb{F}_{q}^{2} \backslash\{(0,0)\}$, we consider

$$
\begin{aligned}
N_{1}(a, b)= & \mid\left\{(x, y) \in \mathbb{F}_{q}^{2} \backslash\{(0,0)\}: \operatorname{Tr}\left(x+y^{p^{u}+1}\right)=0 \text { and } \operatorname{Tr}(a x+b y)=0\right\} \mid \\
= & \sum_{x, y \in \mathbb{F}_{q}}\left(\frac{1}{p} \sum_{z_{1} \in \mathbb{F}_{p}} \zeta_{p}^{z_{1} \operatorname{Tr}\left(x+y^{p^{u}+1}\right)}\right)\left(\frac{1}{p} \sum_{z_{2} \in \mathbb{F}_{p}} \zeta_{p}^{z_{2} \operatorname{Tr}(a x+b y)}\right)-1 \\
= & \frac{1}{p^{2}} \sum_{x, y \in \mathbb{F}_{q}}\left(1+\sum_{z_{1} \in \mathbb{F}_{p}^{*}} \zeta_{p}^{z_{1} \operatorname{Tr}\left(x+y^{p^{u}+1}\right)}\right)\left(1+\sum_{z_{2} \in \mathbb{F}_{p}^{*}} \zeta_{p}^{\left.z_{2} \operatorname{Tr}(a x+b y)\right)-1}\right) \\
= & p^{2 m-2}-1+\frac{1}{p^{2}}\left(\sum_{z_{1} \in \mathbb{F}_{p}^{*}} \sum_{x \in \mathbb{F}_{q}} \zeta_{p}^{\operatorname{Tr}\left(z_{1} x\right)} S_{u}\left(z_{1}, 0\right)+\right. \\
& \sum_{z_{2} \in \mathbb{F}_{p}^{*} x \in \mathbb{F}_{q}} \zeta_{p}^{\operatorname{Tr}\left(z_{2} a x\right)} \sum_{y \in \mathbb{F}_{q}} \zeta_{p}^{\operatorname{Tr}\left(z_{2} b y\right)}+\sum_{z_{1}, z_{2} \in \mathbb{F}_{p}^{*}} \sum_{x \in \mathbb{F}_{q}} \zeta_{p}^{\operatorname{Tr}\left(\left(z_{1}+z_{2} a\right) x\right)} S_{u}\left(z_{1}, z_{2} b\right) \\
= & \begin{cases}p^{2 m-2}-1, & \text { if } a \notin \mathbb{F}_{p}^{*}, \\
p^{2 m-2}-1+p^{m-2} \Omega, & \text { if } a \in \mathbb{F}_{p}^{*},\end{cases}
\end{aligned}
$$

where

$$
\Omega=\sum_{z_{2} \in \mathbb{F}_{p}^{*}} S_{u}\left(-z_{2} a, z_{2} b\right),
$$

and it's clear that

$$
W_{H}(c(a, b))=n_{1}-N_{1}(a, b) .
$$


If $m$ is odd, by Lemma 2 and 6

$$
\begin{aligned}
\Omega & =G(\eta) \sum_{z_{2} \in \mathbb{F}_{p}^{*}} \eta\left(-z_{2} a\right) \bar{\chi}\left(-z_{2} a \gamma_{a, b}^{p^{u}+1}\right) \\
& =G(\eta) \sum_{z_{2} \in \mathbb{F}_{p}^{*}} \eta_{p}\left(-z_{2} a\right) \zeta_{p}^{z_{2} a \operatorname{Tr}\left(\gamma_{a, b}^{p^{u}+1}\right)} \\
& = \begin{cases}0, & \text { if } \operatorname{Tr}\left(\gamma_{a, b}^{p^{u}+1}\right)=0 \\
G(\eta) G\left(\eta_{p}\right) \eta_{p}\left(-\operatorname{Tr}\left(\gamma_{a, b}^{p^{u}+1}\right)\right), & \text { if } \operatorname{Tr}\left(\gamma_{a, b}^{p^{u}+1}\right) \neq 0 .\end{cases}
\end{aligned}
$$

By Lemma 1

$$
G(\eta) G\left(\eta_{p}\right)=\left(p^{*}\right)^{\frac{m+1}{2}} .
$$

By (8), (9), (10) and (11) we know that the three nonzero weights of $C_{D_{1}}$ are $w_{1}=$ $(p-1) p^{2 m-2}, w_{2}=(p-1) p^{2 m-2}\left(1-\frac{1}{(p-1) p^{\frac{m-1}{2}}}\right), w_{3}=(p-1) p^{2 m-2}\left(1+\frac{1}{(p-1) p^{\frac{m-1}{2}}}\right)$ and by Lemma 12, $A_{w_{2}}+A_{w_{3}}=\mid\left\{(a, b) \in \mathbb{F}_{q}^{2}: a \in \mathbb{F}_{p}^{*}\right.$ and $\left.\operatorname{Tr}\left(\gamma_{a, b}^{p^{u}+1}\right) \neq 0\right\} \mid=$ $(p-1)\left(q-p^{m-1}\right)$.

If $\frac{m}{v}$ is odd and $v$ is even, by Lemma 2 and 6

$$
\begin{aligned}
\Omega & =G(\eta) \sum_{z_{2} \in \mathbb{F}_{p}^{*}} \eta\left(-z_{2} a\right) \bar{\chi}\left(-z_{2} a \gamma_{a, b}^{p^{u}+1}\right) \\
& =G(\eta) \sum_{z_{2} \in \mathbb{F}_{p}^{*}} \zeta_{p}^{z_{2} a \operatorname{Tr}\left(\gamma_{a, b}^{p^{u}+1}\right)} \\
& = \begin{cases}(p-1) G(\eta), & \text { if } \operatorname{Tr}\left(\gamma_{a, b}^{p^{u}+1}\right)=0 \\
-G(\eta), & \text { if } \operatorname{Tr}\left(\gamma_{a, b}^{p^{u}+1}\right) \neq 0 .\end{cases}
\end{aligned}
$$

By Lemma 1] $|G(\eta)|=p^{s}$. By (8), (9) and (12) we know that the three nonzero weights of $C_{D_{1}}$ are $w_{1}=(p-1) p^{2 m-2}, w_{2}=(p-1) p^{2 m-2}\left(1-\frac{G(\eta)}{q}\right), w_{3}=$ $(p-1) p^{2 m-2}\left(1+\frac{G(\eta)}{(p-1) q}\right)$ and $A_{w_{2}}+A_{w_{3}}=\left|\left\{(a, b) \in \mathbb{F}_{q}^{2}: a \in \mathbb{F}_{p}^{*}\right\}\right|=(p-1) q$.

If $\frac{m}{v} \equiv 2(\bmod 4)$, by Lemma 6

$$
\begin{aligned}
\Omega & =-p^{s} \sum_{z_{2} \in F_{p}^{*}} \bar{\chi}\left(-z_{2} a \gamma_{a, b}^{p^{u}+1}\right) \\
& = \begin{cases}-(p-1) p^{s}, & \text { if } \operatorname{Tr}\left(\gamma_{a, b}^{p^{u}+1}\right)=0, \\
p^{s}, & \text { if } \operatorname{Tr}\left(\gamma_{a, b}^{p^{u}+1}\right) \neq 0 .\end{cases}
\end{aligned}
$$

By (8), (9) and (13) we know that the three nonzero weights of $C_{D_{1}}$ are $w_{1}=$ $(p-1) p^{2 m-2}, w_{2}=(p-1) p^{2 m-2}\left(1+\frac{1}{p^{s}}\right), w_{3}=(p-1) p^{2 m-2}\left(1-\frac{1}{(p-1) p^{s}}\right)$ and $A_{w_{2}}+A_{w_{3}}=\left|\left\{(a, b) \in \mathbb{F}_{q}^{2}: a \in \mathbb{F}_{p}^{*}\right\}\right|=(p-1) q$.

If $\frac{m}{v} \equiv 0(\bmod 4)$, by Lemma 7

$$
\Omega=0
$$


or (

$$
\begin{aligned}
\Omega & =-p^{s+v} \sum_{z_{2} \in \mathbb{F}_{p}^{*}} \bar{\chi}\left(-z_{2} a \gamma_{a, b}^{p^{u}+1}\right) \\
& = \begin{cases}-(p-1) p^{s+v}, & \text { if } \operatorname{Tr}\left(\gamma_{a, b}^{p^{u}+1}\right)=0, \\
p^{s+v}, & \text { if } \operatorname{Tr}\left(\gamma_{a, b}^{p^{u}+1}\right) \neq 0 .\end{cases}
\end{aligned}
$$

By (8), (9), (14) and (15) we know that the three nonzero weights of $C_{D_{1}}$ are $w_{1}=$ $(p-1) p^{2 m-2}, w_{2}=(p-1) p^{2 m-2}\left(1+\frac{1}{p^{s-v}}\right), w_{3}=(p-1) p^{2 m-2}\left(1-\frac{1}{(p-1) p^{s-v}}\right)$ and by Lemma 13. $A_{w_{2}}+A_{w_{3}}=\mid\left\{(a, b) \in \mathbb{F}_{q}^{2}: a \in \mathbb{F}_{p}^{*}\right.$ and (E1) is solvable over $\left.\mathbb{F}_{q}\right\} \mid=$ $p^{m-2 v}(p-1)$.

The weight distribution of $C_{D_{1}}$ follows from the result of $A_{w_{2}}+A_{w_{3}}$ and the first two Pless power moments.

4.3 The proofs of Theorems 5 and 6

The proofs are similar to previous theorems. The length of $C_{D_{2}}$ is given in Lemma 11. For $(a, b) \in \mathbb{F}_{q}^{2} \backslash\{(0,0)\}$, we consider

$$
\begin{aligned}
N_{2}(a, b) & =\mid\left\{(x, y) \in \mathbb{F}_{q}^{2} \backslash\{(0,0)\}: \operatorname{Tr}\left(x^{2}+y^{p^{u}+1}\right)=0 \text { and } \operatorname{Tr}(a x+b y)=0\right\} \mid \\
& =\sum_{x, y \in \mathbb{F}_{q}}\left(\frac{1}{p} \sum_{z_{1} \in \mathbb{F}_{p}} \zeta_{p}^{z_{1} \operatorname{Tr}\left(x^{2}+y^{p^{u}+1}\right)}\right)\left(\frac{1}{p} \sum_{z_{2} \in \mathbb{F}_{p}} \zeta_{p}^{z_{2} \operatorname{Tr}(a x+b y)}\right)-1 \\
& =p^{2 m-2}-1+\frac{1}{p^{2}}\left(\Omega_{1}+\Omega_{2}\right),
\end{aligned}
$$

where

$$
\Omega_{1}=\sum_{z_{1} \in \mathbb{F}_{p}^{*}} Q\left(z_{1}, 0\right) S_{u}\left(z_{1}, 0\right)
$$

and

$$
\Omega_{2}=\sum_{z_{1}, z_{2} \in \mathbb{F}_{p}^{*}} Q\left(z_{1}, z_{2} a\right) S_{u}\left(z_{1}, z_{2} b\right) .
$$

Note that we have discussed $\Omega_{1}$ in Lemma 11 Let $\gamma_{b} \in \mathbb{F}_{q}$ be some solution of the equation

$$
X^{p^{2 u}}+X=-b^{p^{u}}
$$

if it exists, then for $z_{1}, z_{2} \in \mathbb{F}_{p}^{*}, z_{3} \gamma_{b}$ is the solution of $z_{1}^{p^{u}} X^{p^{2 u}}+z_{1} X=-\left(z_{2} b\right)^{p^{u}}$, where $z_{3}=z_{1}^{-1} z_{2}$.

By Lemma 2 and 8 if $\frac{m}{v}$ is odd,

$$
\begin{aligned}
\Omega_{2} & =G(\eta)^{2} \sum_{z_{1}, z_{3} \in \mathbb{F}_{p}^{*}} \bar{\chi}\left(\frac{z_{1} z_{3}^{2} a^{2}}{4}+z_{1}\left(z_{3} \gamma_{b}\right)^{p^{u}+1}\right) \\
& =G(\eta)^{2} \sum_{z_{1}, z_{3} \in \mathbb{F}_{p}^{*}} \zeta_{p}^{-z_{1} z_{3}^{2} \operatorname{Tr}\left(\frac{a^{2}}{4}+\gamma_{b}^{p^{u}+1}\right)} \\
& = \begin{cases}(p-1)^{2} G(\eta)^{2}, & \text { if } \operatorname{Tr}\left(\frac{a^{2}}{4}+\gamma_{b}^{p^{u}+1}\right)=0, \\
-(p-1) G(\eta)^{2}, & \text { if } \operatorname{Tr}\left(\frac{a^{2}}{4}+\gamma_{b}^{p^{u}+1}\right) \neq 0 .\end{cases}
\end{aligned}
$$


And if $\frac{m}{v} \equiv 2(\bmod 4)$,

$$
\begin{aligned}
\Omega_{2} & =-p^{s} G(\eta) \sum_{z_{1}, z_{3} \in \mathbb{F}_{p}^{*}} \eta\left(z_{1}\right) \bar{\chi}\left(\frac{z_{1} z_{3}^{2} a^{2}}{4}+z_{1}\left(z_{3} \gamma_{b}\right)^{p^{u}+1}\right) \\
& =-p^{s} G(\eta) \sum_{z_{1}, z_{3} \in \mathbb{F}_{p}^{*}} \zeta_{p}^{-z_{1} z_{3}^{2} \operatorname{Tr}\left(\frac{a^{2}}{4}+\gamma_{b}^{p^{u}+1}\right)} \\
& = \begin{cases}-(p-1)^{2} p^{s} G(\eta), & \text { if } \operatorname{Tr}\left(\frac{a^{2}}{4}+\gamma_{b}^{p^{u}+1}\right)=0, \\
(p-1) p^{s} G(\eta), & \text { if } \operatorname{Tr}\left(\frac{a^{2}}{4}+\gamma_{b}^{p^{u}+1}\right) \neq 0 .\end{cases}
\end{aligned}
$$

From the proof of Lemma 11 and (16), (17), (18), (19), (20) we know that the two nonzero weights of $C_{D_{2}}$ are $w_{1}=(p-1) p^{2 m-2}$ and

$$
w_{2}= \begin{cases}(p-1) p^{2 m-2}\left(1-\frac{1}{p^{m-1}}\right), & \text { if } p \equiv 3 \quad(\bmod 4) \text { and } v \text { is odd } \\ (p-1) p^{2 m-2}\left(1+\frac{1}{p^{m-1}}\right), & \text { otherwise }\end{cases}
$$

If $\frac{m}{v} \equiv 0(\bmod 4)$, by Lemma 1, 2 , 7 and 8

$$
\Omega_{2}=0
$$

or ( $(\mathrm{E} 2)$ is solvable $)$

$$
\begin{aligned}
& \Omega_{2}=-p^{s+v} G(\eta) \sum_{z_{1}, z_{3} \in \mathbb{F}_{p}^{*}} \eta\left(z_{1}\right) \bar{\chi}\left(\frac{z_{1} z_{3}^{2} a^{2}}{4}+z_{1}\left(z_{3} \gamma_{b}\right)^{p^{u}+1}\right) \\
& =p^{m+v} \sum_{z_{1}, z_{3} \in \mathbb{F}_{p}^{*}} \zeta_{p}^{-z_{1} z_{3}^{2} \operatorname{Tr}\left(\frac{a^{2}}{4}+\gamma_{b}^{p^{u}+1}\right)} \\
& = \begin{cases}(p-1)^{2} p^{m+v}, & \text { if } \operatorname{Tr}\left(\frac{a^{2}}{4}+\gamma_{b}^{p^{u}+1}\right)=0 \\
-(p-1) p^{m+v}, & \text { if } \operatorname{Tr}\left(\frac{a^{2}}{4}+\gamma_{b}^{p^{u}+1}\right) \neq 0\end{cases}
\end{aligned}
$$

By (16), (17), (18), (17), (21) and (22) we know that the three nonzero weights of $C_{D_{2}}$ are $w_{1}=(p-1) p^{2 m-2}, w_{2}=(p-1) p^{2 m-2}\left(1+\frac{p-1}{p^{m-v}}\right), w_{3}=(p-1) p^{2 m-2}\left(1+\frac{1}{p^{m-v-1}}\right)$ and by Lemma 13, $A_{w_{2}}=\mid\left\{(a, b) \in \mathbb{F}_{q}^{2} \backslash\{(0,0)\}:\right.$ (E2) has no solution over $\left.\mathbb{F}_{q}\right\} \mid=$ $q\left(q-p^{m-2 v}\right)$.

The weight distribution of $C_{D_{2}}$ follows from the first two Pless power moments.

\section{Conclusions}

In this paper, inspired by the work in [16, several classes of two-weight and threeweight linear codes were constructed with their weight distributions settled using Weil sums. Some optimal or almost optimal linear codes were found. It would be interesting if more linear codes with few weights can be presented. 


\section{References}

1. AR Calderbank and JM Goethals. Three-weight codes and association schemes. Philips Journal of Research, 39(4-5):143-152, 1984.

2. R Calderbank and WM Kantor. The geometry of two-weight codes. Bulletin of the London Mathematical Society, 18(2):97-122, 1986.

3. Claude Carlet, Cunsheng Ding, and Jin Yuan. Linear codes from perfect nonlinear mappings and their secret sharing schemes. IEEE Transactions on Information Theory, 51(6):2089-2102, 2005

4. Robert S Coulter. Explicit evaluations of some Weil sums. Acta Arithmetica, 83:241-251, 1998

5. Robert S Coulter. Further evaluations of Weil sums. Acta Arithmetica, 86:217-226, 1998.

6. Cunsheng Ding and Harald Niederreiter. Cyclotomic linear codes of order 3. IEEE Transactions on Information Theory, 53(6):2274-2277, 2007.

7. Cunsheng Ding and Xuesong Wang. A coding theory construction of new systematic authentication codes. Theoretical Computer Science, 330(1):81-99, 2005

8. Kelan Ding and Cunsheng Ding. Binary linear codes with three weights. IEEE Communications Letters, 18(11):1879-1882, 2014.

9. Kelan Ding and Cunsheng Ding. A class of two-weight and three-weight codes and their applications in secret sharing. IEEE Transactions on Information Theory, 61(11):5835$5842,2015$.

10. James H Griesmer. A bound for error-correcting codes. IBM Journal of Research and Development, 4(5):532-542, 1960.

11. Ziling Heng and Qin Yue. A class of binary linear codes with at most three weights. IEEE Communications Letters, 19(9):1488-1491, 2015.

12. Ziling Heng and Qin Yue. Two classes of two-weight linear codes. Finite Fields and Their Applications, 38:72-92, 2016.

13. Ziling Heng and Qin Yue. A construction of $q$-ary linear codes with two weights. Finite Fields and Their Applications, 48:20-42, 2017.

14. Ziling Heng, Qin Yue, and Chengju Li. Three classes of linear codes with two or three weights. Discrete Mathematics, 339(11):2832-2847, 2016.

15. W Cary Huffman and Vera Pless. Fundamentals of error-correcting codes. Cambridge university press, 2010.

16. Chengju Li, Qin Yue, and FangWei Fu. A construction of several classes of two-weight and three-weight linear codes. Applicable Algebra in Engineering, Communication and Computing, pages 1-20, 2016.

17. Rudolf Lidl and Harald Niederreiter. Finite fields, volume 20. Cambridge university press, 1997.

18. Gaojun Luo, Xiwang Cao, Shanding Xu, and Jiafu Mi. Binary linear codes with two or three weights from niho exponents. Cryptography and Communications, 10(2):301-318, 2018

19. Chunming Tang, Nian Li, Yanfeng Qi, Zhengchun Zhou, and Tor Helleseth. Linear codes with two or three weights from weakly regular bent functions. IEEE Transactions on Information Theory, 62(3):1166-1176, 2016.

20. Klove Torleiv. Codes For Error Detection, volume 2. World Scientific, 2007.

21. Jin Yuan and Cunsheng Ding. Secret sharing schemes from three classes of linear codes. IEEE Transactions on Information Theory, 52(1):206-212, 2006.

22. Zhengchun Zhou, Nian Li, Cuiling Fan, and Tor Helleseth. Linear codes with two or three weights from quadratic bent functions. Designs, Codes and Cryptography, pages 1-13, 2015 